\title{
Solitary cystic dilatation of the intrahepatic bile duct
}

\author{
K Ohmoto, M Shimizu, Y Iguchi, S Yamamoto, M Murakami, T Tsunoda
}

Division of

Gastroenterology,

Department of

Medicine, Kawasaki

Medical School,

Kurashiki, Japan

K Ohmoto

Y Iguchi

S Yamamoto

Department of

Pathology

M Shimizu

Department of

Surgery

M Murakami

T Tsunoda

Correspondence to: Dr Ohmoto, Division of Gastroenterology,

Department of Medicine,

Kawasaki Medical School,

577 Matsushima, Kurashiki,

Okayama 701-01, Japan.

Accepted for publication 11 March 1997

\begin{abstract}
A 31 year old man was hospitalised with general fatigue and epigastric pain. Abdominal ultrasonography, computed tomography, and magnetic resonance imaging showed a cystic lesion in the left lobe of the liver. Endoscopic retrograde cholangiopancreatography and percutaneous transhepatic cholangiography revealed a localised dilatation of the intrahepatic bile duct without any obstruction. However, a large mass of mucinous material was noted in the saccular intrahepatic duct and the common bile duct. There was no evidence of a choledochal cyst, anomalous pancreaticobiliary ductal union, or congenital cystic change of the kidneys. A possible diagnosis of mucinous cystic neoplasm of the intrahepatic bile duct was made and a left hepatectomy performed. Cholangiography of the resected specimen showed a non-obstructive solitary cystic dilatation of the left hepatic duct. Histologically, the dilated duct was lined with columnar biliary epithelium without any papillary proliferation and/or atypia. Neither malignancy nor hepatic fibrosis was observed; the term "solitary cystic dilatation of the intrahepatic bile duct" perhaps is more descriptive and the concept easier to understand.

(f Clin Pathol 1997;50:617-618)
\end{abstract}

Keywords: solitary cystic dilatation; intrahepatic bile duct; terminology

Recently, Terada and Nakanuma described two cases of solitary cystic dilatation of the intrahepatic bile duct without choledochal cysts, anomalous pancreaticobiliary ductal union, or congenital cystic change in the kidneys. ${ }^{1}$ To date, only 13 such cases have been reported. ${ }^{1-6}$ Several other terms have been used in the literature with regard to localised or non-obstructive dilatation of the intrahepatic bile duct. These include the localised form of Caroli's disease, ${ }^{78}$ localised dilatation of a major intrahepatic duct, ${ }^{4}$ and a type V congenital bile duct cyst. ${ }^{9}$

We report a case of solitary cystic dilatation of the intrahepatic bile duct as well as a review of the literature, and discuss the terminology concerning this entity.

\section{Case report}

In April 1995, a 31 year old man was admitted to Kawasaki Medical School Hospital because of general fatigue and epigastric pain. $\mathrm{He}$ presented with neither a pertinent family history nor a personal history of blood transfusion, tattooing, or drug abuse. On physical examination, there was a slight tenderness in the epigastrium and right hypochondrium, and the liver was slightly enlarged. Results of liver function tests were: total bilirubin, $2.1 \mathrm{mg} / 1$ (normal value $0.2-1.0$ ); alkaline phosphate, 127 IU/1 (28-84); $\gamma$ glutamyltranspeptidase $314 \mathrm{IU} / 1$ (4-30); aspartate aminotransferase $117 \mathrm{IU} / 1$ (7-20); and alanine aminotransferase $425 \mathrm{IU} / 1$ (7-28). Virus markers were negative for hepatitis A, B, and C. Abdominal ultrasonography, computed tomography, and magnetic resonance imaging showed a cystic lesion in the left lobe of the liver. Choledochal cysts and anomalous pancreaticobiliary ductal union were not demonstrated on endoscopic retrograde cholangiopancreatography. Percutaneous transhepatic cholangiography revealed a localised dilatation of the left intrahepatic bile duct. There was a large mass of mucinous material in the saccular intrahepatic bile ducts and the common bile duct. Under the diagnosis of a possible mucinous cystic neoplasm of the intrahepatic bile duct, we performed a left hepatectomy. A cholangiogram of the resected specimen showed a solitary cystic dilatation of the left hepatic duct (fig 1). Histologically, the

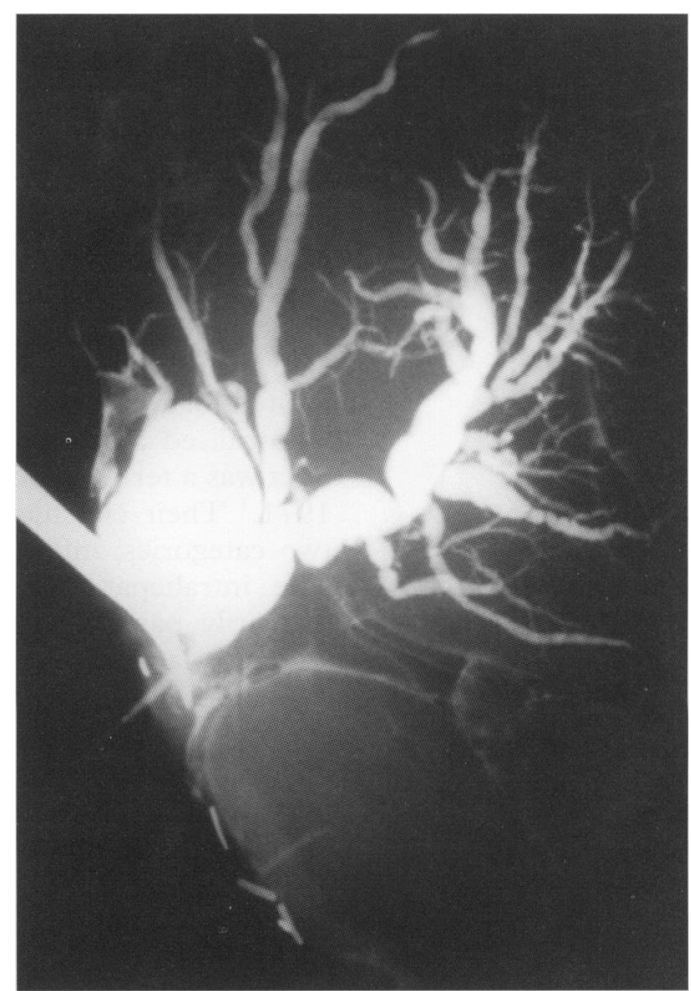

Figure 1 Cholangiogram of the resected specimen showing a solitary cystic dilatation of the left hepatic duct. 


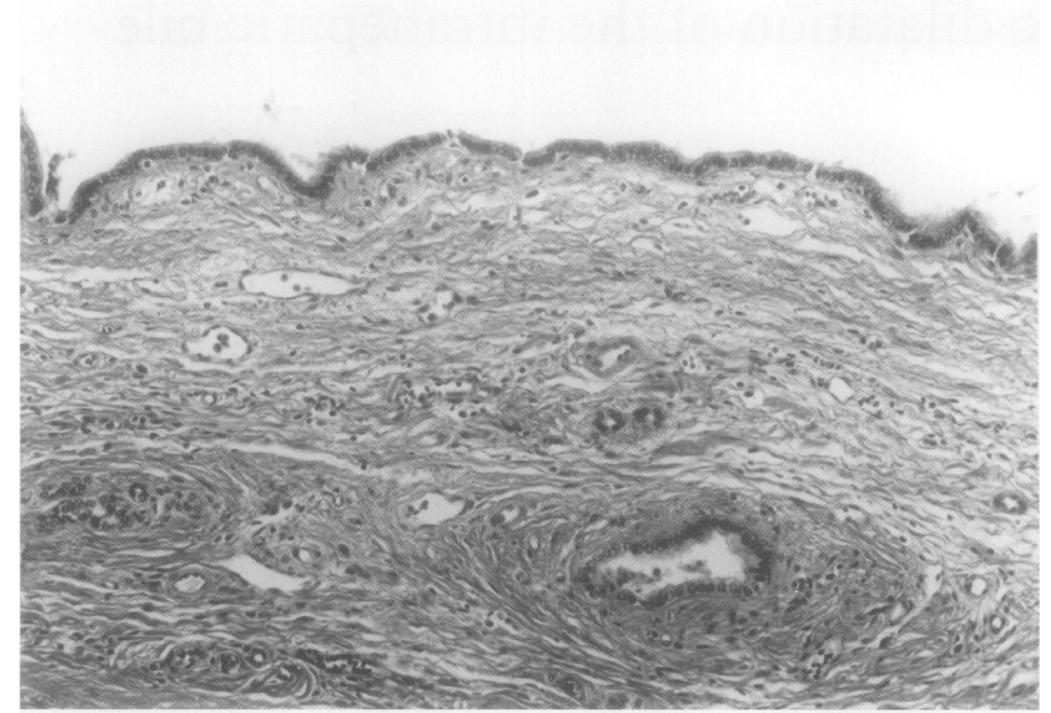

Figure 2 The dilated left hepatic duct is lined with mucinous columnar epithelium (haematoxylin and eosin, original magnification $\times 40$ ).

dilated duct was lined with columnar biliary epithelium without any papillary proliferation or atypia (fig 2). There was no evidence of "mesenchymal stroma", malignancy, or hepatic fibrosis. From these clinical and pathological findings, we made a diagnosis of solitary cystic dilatation of the intrahepatic bile duct.

\section{Discussion}

The terminology concerning localised or nonobstructive dilatation of the intrahepatic bile duct is somewhat controversial. We found that at least four terms have been used in the literature for cases such as ours: localised form of Caroli's disease, ${ }^{7}$ localised dilatation of a major intrahepatic duct, ${ }^{4}$ a type $\mathrm{V}$ congenital bile duct cyst, ${ }^{9}$ and solitary cystic dilatation of the intrahepatic bile duct.'

Caroli's disease, first described by Caroli et al in $1958,{ }^{7}$ is a rare congenital disorder characterised by cystic dilatation of the intrahepatic bile ducts. According to Barros et $a l^{10}$ six of 46 cases $(13 \%)$ of Caroli's disease were isolated forms of intrahepatic cystic dilatations. Our case may be categorised into the localised form of Caroli's disease. However, normally the localised form of Caroli's disease involves all of the left or right lobe of the liver. ${ }^{8}$ Therefore, our case is not a typical case and may be called a limited form of Caroli's disease.

Localised dilatation of a major intrahepatic duct was a term proposed by Longmire et al in 1971. ${ }^{4}$ Their classification is subdivided into two categories: intrahepatic and extrahepatic. The intrahepatic type is also divided into primarily parenchymal and primarily ductal, with the latter being classified into localised dilatation of a major intrahepatic duct and multiple cystic dilatations of the intrahepatic ducts.
The term type V congenital bile duct cyst was proposed by Todani et al in $1977 .^{9}$ Type V is an intrahepatic bile duct cyst and it may be single or multiple (our case was single). Todani et al suggested that type $\mathrm{V}$ might be closely related to intrahepatic stones and could be categorised as a pure form of Caroli's disease without hepatic fibrosis.

Finally, solitary cystic dilatation of the intrahepatic bile duct is a term that was proposed by Terada and Nakanuma in $1987 .{ }^{1}$ Although this is a rather descriptive term, this entity has some clinicopathological features such as female predominance and cystic dilatation involving the right or left hepatic duct near the hepatic hilus. In addition, there is no evidence of related conditions including choledochal cysts, anomalous pancreaticobiliary ductal union, or congenital cystic change in the kidney. As Terada and Nakamura pointed out, bile stasis and mucous hypersecretion are important for dilatation of the intrahepatic bile duct, ${ }^{1}$ our case may be explained by mucous hypersecretion as a large mass of mucinous material was seen in the intrahepatic duct.

To date, the above mentioned terms have been used for the same localised or nonobstructive dilated lesion of the intrahepatic bile duct. We prefer to use the last termsolitary cystic dilatation of the intrahepatic bile duct-because it is descriptive and the concept is easy to understand. Furthermore, it has clinicopathological features. Although this lesion is very rare and under-recognised, it should be considered by both pathologists and clinicians.

1 Terada T, Nakanuma Y. Solitary cystic dilation of the intrahepatic bile duct: morphology of two autopsy cases and review of the literature. Am $\mathcal{F}$ Gastroenterol 1987;82:1301-5.

2 Glenn F, Moody FG. Intrahepatic calculi. Ann Surg 1961;153:711-24.

3 Warren KW, Kune GA, Hardy KJ. Biliary duct cysts. Surg Clin North Am 1968;48:567-77.

4 Longmire WP, Mandiola SA, Gordon HE. Congenital cystic disease of the liver and biliary system. Ann Surg 1971;174: 711-26.

5 Rangabashyam N, Krishnaraj B, Mohan C, Ramakrishnan V. Intrahepatic choledochal cyst. $A m \mathcal{F}$ Gastroenterol 1975;63:71-4

6 Austin RM, Sussman S, McArdle CR, Kim D, Elboim C Case report: computed tomographic and ultrasound appearances of a solitary intrahepatic choledochal cyst. Clin Radiol 1986;37:149-50.

7 Caroli J, Soupault R, Kossakowski J, Plocker L, Paradowska $M$. La dilatation polykystique congénitale des voies biliaire intra-hépatiques. Essai de classification. Sem Hôp Paris 1958;34:488-95.

8 Mercadier M, Chigot JP, Clot JP, Langlois P, Lansiaux P. Caroli's disease. World 7 Surg 1984;8:22-9.

9 Todani T, Watanabe Y, Narusue M, Tabuchi K, Okajima K. Congenital bile duct cysts. Classification, operative procedures, and review of thirty-seven cases including cancer arising from choledochal cyst. Am f Surg 1977;134:263-9.

10 Barros JL, Polo JR, Sanabia J, Garcia-Sabrido JL, GomezLorenzo FJ. Congenital cystic dilatation of the intrahepatic bile ducts (Caroli's disease): report of a case and review of the literature. Surgery 1979;85:589-92. 